\title{
Association of Celiac Disease With Idiopathic Pulmonary Hemosiderosis; Lane Hamilton Syndrome
}

\author{
Hikmet Tekin Nacaroglu, ${ }^{1,}$ Ozlem Sarac Sandal, ${ }^{1}$ Ozlem Bag, ${ }^{2}$ Semiha Bahceci Erdem, ${ }^{1}$ \\ Ozlem Bekem Soylu, ${ }^{3}$ Gulden Diniz, ${ }^{4}$ Aysel Ozturk, ${ }^{2}$ and Demet Can ${ }^{1}$ \\ ${ }^{1}$ Department of Pediatric Allergy, Dr. Behcet Uz Children's Hospital, Izmir, Turkey \\ ${ }^{2}$ Clinics of Pediatrics, Dr. Behcet Uz Children's Hospital, Izmir, Turkey \\ ${ }^{3}$ Department of Pediatric Gastroenterology, Dr. Behcet Uz Children's Hospital, Izmir, Turkey \\ ${ }^{4}$ Department of Pathology, Dr. Behcet Uz Children's Hospital, Izmir, Turkey \\ ${ }^{*}$ Corresponding Author: Hikmet Tekin Nacaroglu, Department of Pediatric Allergy, Dr. Behcet Uz Children’s Hospital, Izmir, Turkey. Tel: +90-2324116319, Fax: +90-2324892315, \\ E-mail: tekin212@gmail.com
}

Received: July 2, 2015; Accepted: August 9, 2015

\begin{abstract}
Introduction: Idiopathic Pulmonary Hemosiderosis (IPH) is a rare cause of alveolar hemorrhage, which is seen primarily in childhood. Celiac disease is defined as a chronic, immune-mediated enteropathy of the small intestine, caused by exposure to dietary gluten in genetically pre-disposed individuals. Association of IPH and celiac disease is known as Lane Hamilton syndrome. There are limited number of case reports of this syndrome in literature.

Case Presentation: Although there were no growth and developmental delay and gastrointestinal symptoms like chronic diarrhea, chronic constipation, vomiting, abdominal bloating and pain in the two patients with IPH, they were diagnosed with Lane Hamilton Syndrome. After initiation of gluten-free diet, their IPH symptoms disappeared and hemoglobin levels were observed to return to normal. Conclusions: Even if there were no gastrointestinal symptoms in a patient with IPH, celiac disease should be investigated. These patients may benefit from gluten free diet and IPH symptoms may disappear.
\end{abstract}

Keywords: Idiopathic Pulmonary Hemosiderosis; Celiac Disease; Lane-Hamilton Syndrome; Gluten-Free Diet

\section{Introduction}

Idiopathic pulmonary hemosiderosis (IPH), primarily seen in childhood, is an uncommon cause of alveolar hemorrhage with an unknown pathogenesis. It is clinically characterized by diffuse parenchymal infiltrates, hemoptysis and secondary iron deficiency anemia. A definitve diagnosis can be established with the presentation of macrophages loaded with hemosiderin in the open lung biopsy or sputum and bronchoalveolar lavage fluid (BLF) (1).

Celiac disease, on the other hand manifests itself with gastrointestinal symptoms in childhood and is an autoimmune enteropathy that appears with the ingestion of foods including gluten in individuals with genetic predisposition (2). Association of IPH and celiac disease is called as Lane Hamilton syndrome and is somewhat a rarely seen condition (3). There are limited number of case reports of this syndrome in literature. This so-called association must be searched since gluten-free diet can reduce corticosteroid requirement in the patients.

\section{Case Presentation}

\subsection{Case I}

A 16 year old male patient was admitted to our hospital with weakness, rapid exhaustion and bloody sputum since six months ago. He was on iron supplement therapy due to anemia. Parents were close relatives and healthy and family history was negative for similar symptoms. His weight was $58.5 \mathrm{~kg}$ (25 - 50 percentile) and his height was $174 \mathrm{~cm}$ ( 50 - 75 percentile). His systemic examination was unremarkable. Laboratory data were: $\mathrm{Hb} 5.8$ g/dL, RBC 3,420.000/ $\mathrm{mm}^{3}$, MCV 63, Hct 21.5\%, hypochromic microcytic anemia was detected. Sedimentation rate

Copyright (C) 2015, Growth \& Development Research Center. This is an open-access article distributed under the terms of the Creative Commons Attribution-NonCommercial 4.0 International License (http://creativecommons.org/licenses/by-nc/4.0/) which permits copy and redistribute the material just in noncommercial usages, provided the original work is properly cited. 
was $27 \mathrm{~mm} /$ hour, C-reactive protein $0.33 \mathrm{mg} / \mathrm{dL}$. Serum iron $12 \mu \mathrm{g} / \mathrm{dL}$, total iron-binding capacity (TIBC) $443 \mu \mathrm{g} /$ $\mathrm{dL}$, ferritin $8.57 \mathrm{ng} / \mathrm{mL}$. Peripheral blood smear and bone marrow biopsy were compatible with iron deficiency anemia. No evidence of bleeding diathesis was found, kidney and liver function tests were unremarkable. Increased density was observed in chest X-rays in base of both lungs. In thoracic CT scan there were densities with ground glass appearance in both lungs which were more distinct in posterobasal levels (Figure 1). In the fiberoptic bronchoscopy, endobronchial lesion and bleeding focus were not detected. In the BLF, many hemosiderin laden macrophages were seen. Serum ANA (ELISA), C-ANCA, PANCA, Anti-dsDNA were found negative. C3, C4, IgG, IgA, IgM, IgE, inhaler specific IgE and FX5 nourishment panel values were normal. No atopy was established in skin tests. Cardiac evaluation was normal. Spirometric assessment was as follows: FVC 81\%, FEV1 84\%, FEV1/FVC 100\%, PEF $106 \%$. Though there were no GIS symptoms, Anti Gliadin IgA and IgG and Anti Endomysium antibodies were determined as positive. In endoscopy duodenal pili finding was compatible with celiac disease and in duodenal biopsy total villous atrophy was observed (Figure 2). Gluten free diet was initiated and hemoglobin level returned to normal after sixteen months.

\subsection{Case II}

A 9 year-old male patient was admitted to our clinic with complaints of paleness, weakness and hemoptysis since 3 - 4 days ago. His body weight was $26 \mathrm{~kg}$ (10 percentile) and height was $130 \mathrm{~cm}$ (25-50 percentile). Apart from 1/6 systolic murmur in cardiac examination, no specific sign was determined. The peripheral blood smear was compatible with severe hypochromic microcytic anemia. Bone marrow biopsy revealed active erythropoeisis. Laboratory data are as follow: serum iron $18 \mu \mathrm{g} / \mathrm{dL}$, TIBC $404 \mu \mathrm{g} / \mathrm{dL}$, ferritin $12 \mathrm{ng} / \mathrm{mL}$. The thoracic CT scan revealed areas of higher density with ground glass appearance characterized by extensive alveolar involvement in both lungs. Fiberoptic bronchoscopy was performed and many hemosiderin laden macrophages were found in BAL fluid. Diagnosed with IPH parenteral steroid treatment with a dose of $2 \mathrm{mg} / \mathrm{kg} /$ day was initiated and erythrocyte transfusion support provided. Since the Anti Gliadin IgA and IgG and Anti Endomysium antibodies were found to be positive, GIS endoscopy was performed and the duodenal biopsy revealed MARSH 3B histological findings compatible with celiac disease (Figure 3). No remarkable finding was established in other examinations. Gluten-free diet was commenced for celiac disease. In the follow-up, steroid treatment was tapered gradually and discontinued, gluten-free diet was continued and hemoglobin level returned to normal in nine months.

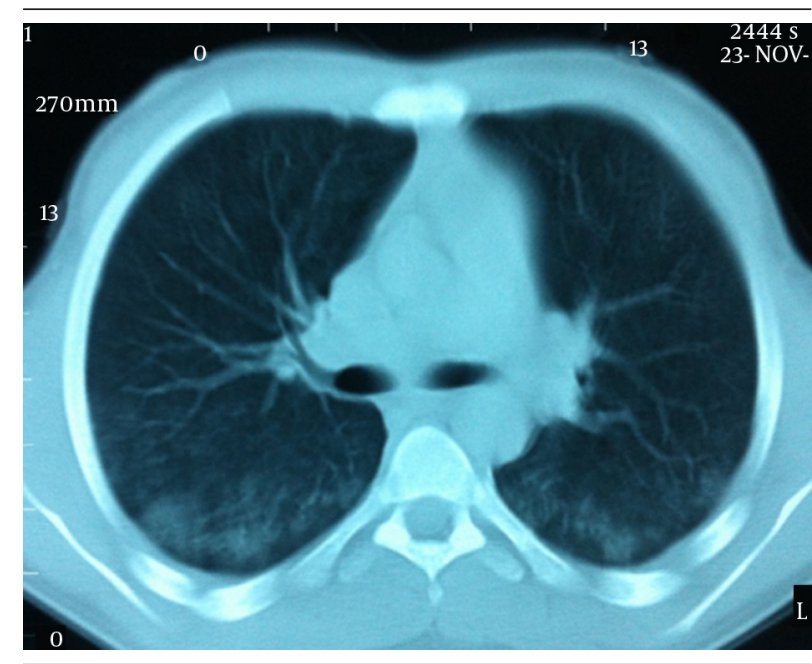

Figure 1. More Distinct Diffuse Ground Glass Densities in Both Lungs at Posterobasal Zones

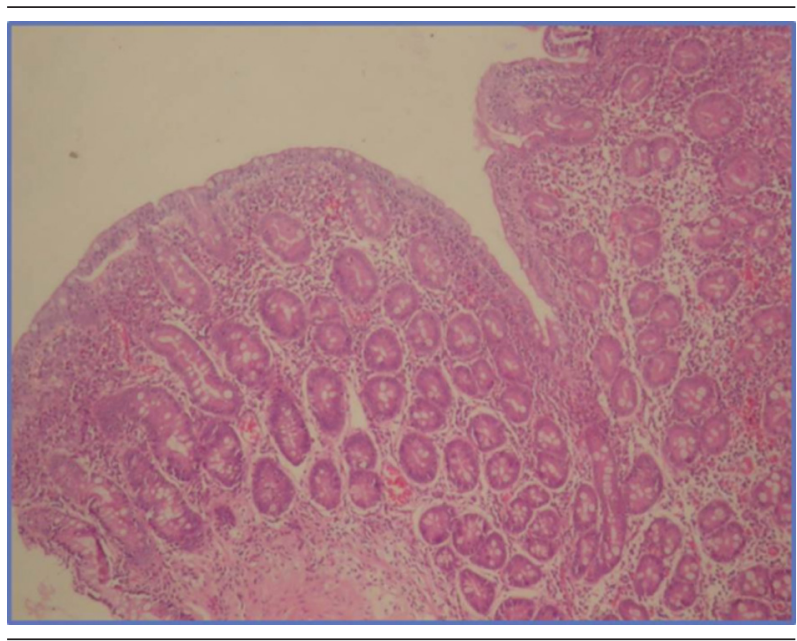

Figure 2. Total Villous Atrophy $(\mathrm{HE} \times 40)$

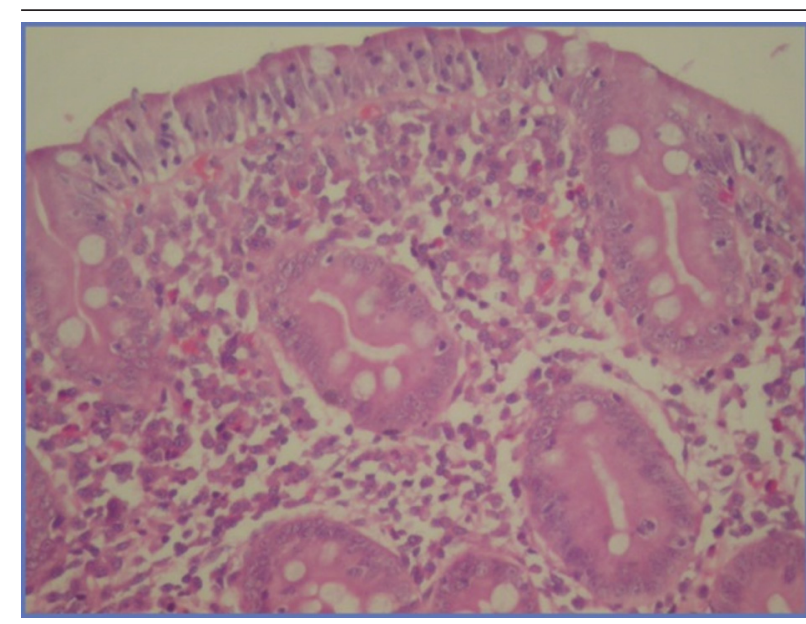

Figure 3. There is a Distinct Flattening in Villi and Intraepithelial Lymphocyte Increase in Histopathological Examination of Duodenal Mucosal Biopsy $(\mathrm{HE} \times 40)$ 


\section{Discussion}

Lane Hamilton syndrome is a very rare situation known since 1971 after Lane and Hamilton described it in a young man for the first time (3). While immunological mechanisms are believed to be effective in the formation of IPH and celiac disease, pathogenic connection between them cannot be precisely understood. CD8 T lymphocytes play a major role in the development of celiac disease. It leads to celiac disease in genetically predisposed individuals through an inappropriate $\mathrm{T}$ cell-mediated immune response stimulated with gluten intake (2). A hypothesis was suggested that there is an alveolar capillary defect in IPH basal membrane or endothelium. It was observed that there was an increase in the ratio of CD8/CD4 when cytological analysis of BAL was carried out in IPH pathogenesis (1). Perelman et al. (4) determined celiac disease in a child with IPH and some hypotheses were asserted regarding this association. The first hypothesis was storage of circulating immune complex involving food allergens in basal membranes of alveolar capillaries. The second was that there was a cross-reactivity between alveolar basal membrane antigen and anti-reticulin antibodies. The third hypothesis was that adenovirus 12, the potential etiological factor of celiac disease, had a role in IPH. Khemiri et al. (5). Recommend serological review for celiac disease only for patients with gastrointestinal symptoms. However, since there was no gastrointestinal system findings related to celiac disease in many reported cases, researchers recommend to perform serologic tests for celiac disease in all cases of $\operatorname{IPH}(4,6,7)$. Some researchers even advise duodenal biopsy to be performed in all patients diagnosed with IPH $(6,7)$. IPH usually responds to corticosteroids well. When IPH is associated with celiac disease, gluten free diet reduces the need for corticosteroids. In some cases, gluten free diet leads to partial or complete regression of symptoms $(6,8,9)$. In 12 out of 16 cases, pulmonary symptoms were observed to get better in patients with gluten free diet (8). Khemiri et al. (5). Underline again the importance of diet by draw- ing attention that pulmonary symptoms show relapse with re-addition of gluten to the diet. In a study involving 3 cases carried out by Sethi et al. (6), it was indicated that pulmonary symptoms recovered completely after gluten-free diet was initiated and immunosuppressive treatments could be decreased in all children. Even after the corticosteroid treatment was stopped, our patients were symptom free with gluten-free diet.

In conclusion, the two cases admitted with hemoptysis and diagnosed with IPH because of the presence of hemosiderin-laden macrophages in BAL in addition to the clinical and radio-logical findings, were finally diagnosed with Lane Hamilton Syndrome even in the absence of gastro-intestinal complaints. We conclude that celiac disease must be investigated in patients diagnosed with IPH despite having no gastrointestinal symptoms. Apparent recovery can be observed with gluten-free diet in IPH.

\section{References}

1. Ioachimescu OC, Sieber S, Kotch A. Idiopathic pulmonary haemosiderosis revisited. Eur Respir J. 2004;24(1):162-70.

2. Hammami S, Ghedira Besbes L, Hadded S, Chouchane S, Ben Meriem C, Gueddiche MN. Co-occurrence pulmonary haemosiderosis with coeliac disease in child. RespirMed. 2008;102(6):935-6.

3. Lane DJ, Hamilton WS. Idiopathic steatorrhoea and idiopathic pulmonary haemosiderosis. Br Med J. 1971;2(5753):89-90.

4. Perelman S, Dupuy C, Bourrillon A. [The association of pulmonary hemosiderosis and celiac disease. Apropos of a new case in a child]. Ann Pediatr (Paris). 1992;39(3):185-8.

5. Khemiri M, Ouederni M, Khaldi F, Barsaoui S. Screening for celiac disease in idiopathic pulmonary hemosiderosis. Gastroenterol Clin Biol. 2008;32(8-9):745-8.

6. Sethi GR, Singhal KK, Puri AS, Mantan M. Benefit of gluten-free diet in idiopathic pulmonary hemosiderosis in association with celiac disease. Pediatr Pulmonol. 2011;46(3):302-5.

7. Keskin O, Keskin M, Guler E, Tutar E, Saygili O, Kucukosmanoglu E, et al. Unusual presentation: pulmonary hemosiderosis with celiac disease and retinitis pigmentosa in a child. Pediatr Pulmonol. 2011;46(8):820-3.

8. Agarwal R, Aggarwal AN, Gupta D. Case 3-2007: a boy with respiratory insufficiency. N Engl J Med. 2007;356(22):2329.

9. Malhotra P, Aggarwal R, Aggarwal AN, Jindal SK, Awasthi A, Radotra BD. Coeliac disease as a cause of unusually severe anaemia in a young man with idiopathic pulmonary haemosiderosis. Respir Med. 2005;99(4):451-3. 